Ran Tao - Buhe Jin · Shen Zheng Guo - Wei Qing

Guo Yin Feng · David G. Brooks · Lijun Liu

Junfu Xu · Taiwei Li - Yujuan Yan - Lin He

\title{
A novel missense mutation of the EDA gene in a Mongolian family with congenital hypodontia
}

Received: 28 October 2005/ Accepted: 30 January 2006/Published online: 1 April 2006

(c) The Japan Society of Human Genetics and Springer-Verlag 2006

\begin{abstract}
X-linked hypohidrotic ectodermal dysplasia (HED) is a rare disease characterized by the hypoplasia or absence of eccrine glands, dry skin, scant hair, and dental abnormalities. Here, we report a Mongolian family with congenital absence of teeth inherited in an $\mathrm{X}$-linked fashion. The affected members of the family did not show other HED characteristics, except hypodontia. We successfully mapped the affected locus to chromosome Xq12-q13.1, and then found a novel missense mutation, c. $193 \mathrm{C}>\mathrm{G}$, in the ectodysplasin A $(E D A)$ gene in all affected males and carrier females. The mutation causes arginine to be replaced by glycine in codon $65(\mathrm{R} 65 \mathrm{G})$ in the juxtamembrane region of $E D A$.
\end{abstract}

Ran Tao, Buhe Jin, Shen Zheng Guo, and Wei Qing contributed equally to this work.

R. Tao $\cdot$ S.Z. Guo $\cdot$ G.Y. Feng

Institute for Nutritional Sciences,

Shanghai Institute of Biological Sciences,

Chinese Academy of Sciences, 294 Taiyuan Road,

200031 Shanghai, China

R. Tao · S.Z. Guo · W. Qing · G.Y. Feng $\cdot$ L. He $(\bowtie)$ Bio-X Life Science Research Center, Shanghai Jiao Tong University, Hao Ran Building, 1954 Hua Shan Road, P.O. Box 501, 200030 Shanghai, China

E-mail: helin@nhgg.org

Tel: + 86-21-62822491

Fax: + 86-21-62822491

B. Jin · D.G. Brooks

Merck Research Laboratories, West Point, PA, USA

L. Liu $\cdot$ J. Xu $\cdot$ T. Li

Department of Internal Medicine, Tongliao Hospital, Tongliao, Neimenggu, China

Y. Yan

Department of Stomatology, Yangpu Central Hospital, Shanghai, China

L. He

Institute for Nutritional Sciences, SIBS, CAS, 319 Yueyang Road, 200031 Shanghai, China
In addition, 33\% (3/9) of female carriers have a skewed $\mathrm{X}$-chromosome inactivation pattern. Our result strongly suggests that the c.193C $>\mathrm{G}$ mutation is the diseasecausing mutation in this family.

Keywords $E D A$ gene $\cdot$ Missense mutation . Oligodontia $\cdot \mathrm{X}$-linked ectodermal dysplasia Xq12-q13.1

\section{Introduction}

Tooth agenesis is a common human anomaly that affects approximately $20 \%$ of the population and is associated with more than 49 syndromes (Pinheiro and Freire-Maia 1994). Hypodontia is agenesis of two or more permanent teeth without associated systemic disorders. The ectodermal structural malformation involving hair, skin, nails, and teeth can be inherited in autosomal dominant, autosomal recessive, or X-linked patterns.

Since Charles Darwin described a peculiar disorder in 1875 (Darwin 1875), many similar cases have been reported and are now referred to as anhidrotic (or hypohidrotic) ectodermal dysplasia (EDA or HED, OMIM 305100). Three associated signs characterize EDA, including sparse hair, abnormal or missing teeth, and inability to sweat due to the lack of sweat glands. Prior studies have determined that mutations of the ectodysplasin A $(E D A)$ gene are responsible for X-linked HED (Kere et al. 1996; Bayes et al. 1998; Monreal et al. 1998). Ectodysplasin, the protein encoded by EDA, is a transmembrane protein member of the tumor necrosis factor family (Ezer et al. 1999). Specific mutations in $E D A$ or its receptor, $E D A R$, result in manifestations of $\mathrm{HED}$ (Monreal et al. 1998; Tucker et al. 2000).

We studied a Mongolian family segregating a unique form of hypodontia in an X-linked recessive manner (Fig. 1a). The affected individuals had normal hair, skin, and nails, but lacked primary and permanent teeth (Fig. 1b). However, the manifestation of hypodontia 


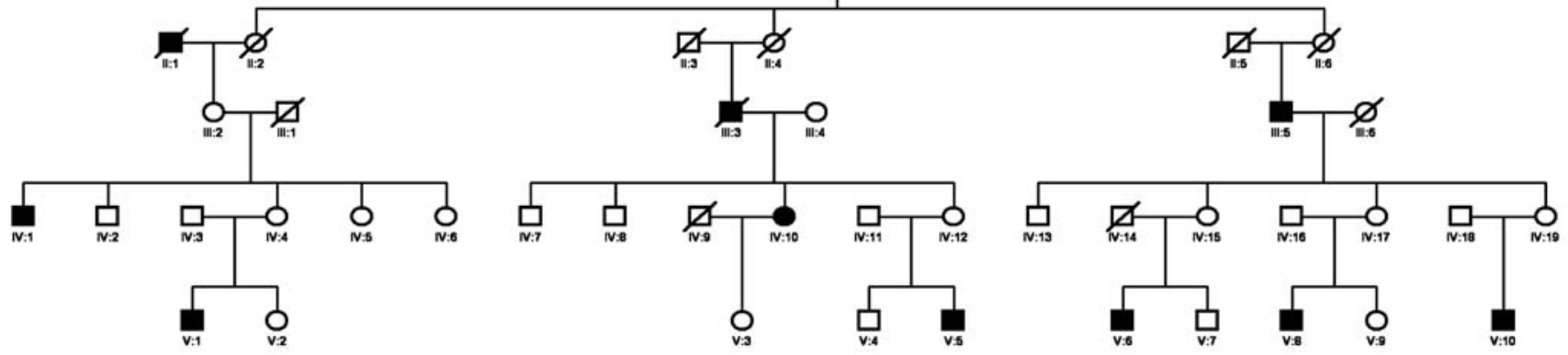

b

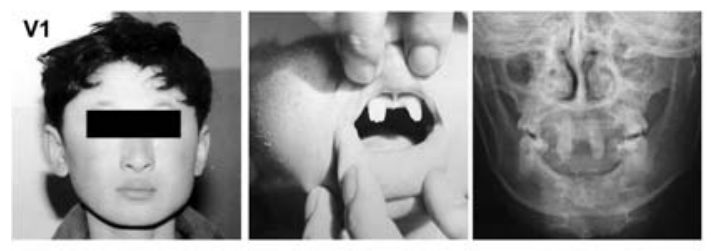

c

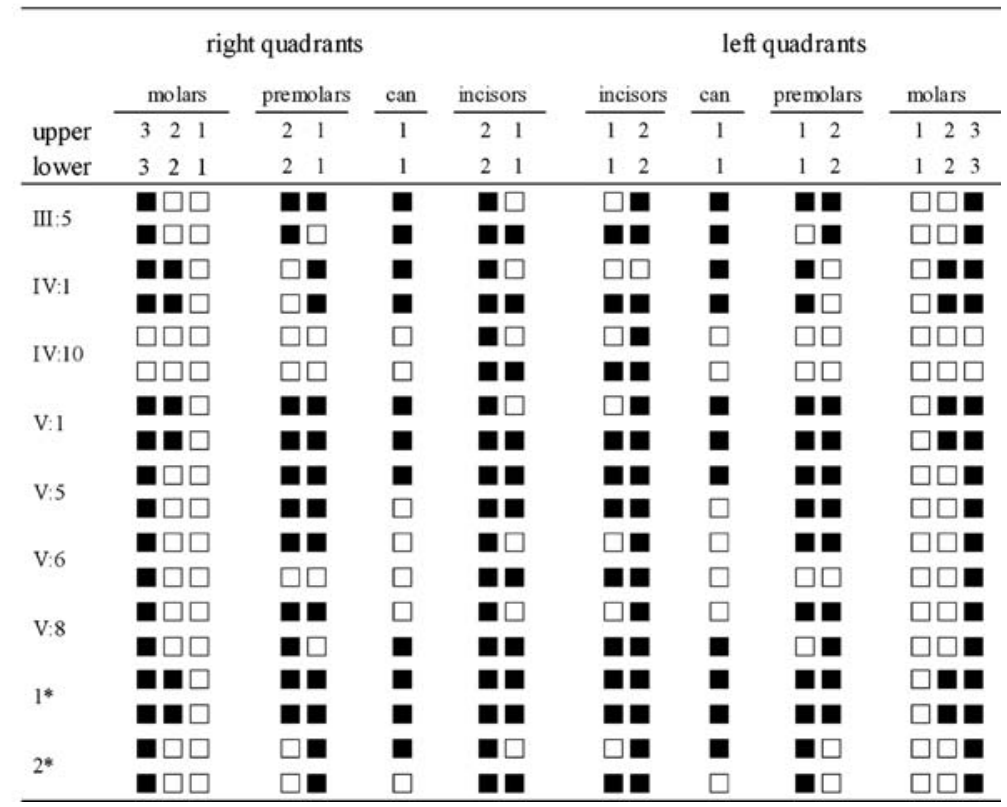

Fig. 1a-c Clinical evaluations. a Pedigree. All affected individuals and carriers have the missense mutation in ectodysplasin $\mathrm{A}(E D A)$. b V:1, 15 years old, has two permanent incisors, four permanent first molars, and four milk molars, but only had two primary incisors and four primary molars in his childhood. c Synopsis of the permanent dentition in affected family members. Filled squares represent absent teeth. Asterisks $\left(^{*}\right)$ represent the affected family members not included in Fig. 2. V:10 in Fig. 2 is too young to be phenotyped in detail and the absence pattern of primary teeth was not listed in detail because of lacking X-ray data is not uniform in this family (Fig. 1c), indicating incomplete penetrance or variable expressivity. The affected members commonly had two pairs of permanent first molars. All of the affected members exhibited congenital absence of their lower incisors and lower lateral incisors.

\section{Subjects and methods}

Informed consents were obtained from all subjects participating in the study. Samples of peripheral blood were taken from 30 available family members for DNA extraction. Linkage analysis employed the Slink simulation program and 50,000 iterations of two-point simulation. By MSIM analysis (tetra $=0.01$ ), the simulation result showed the maximum LOD score was 4.11 and the mean LOD score in this pedigree was 2.76 . Genotyping was performed using microsatellite markers with $10-\mathrm{cM}$ resolution throughout the $\mathrm{X}$ chromosome. Two-point LOD scores were calculated by using the MLINK program of the LINKAGE version 5.1 software package. For understanding the pattern of $\mathrm{X}$-chromosome inactivation, undigested DNA and HpaII-digested DNA were used as a template to detect the androgen-receptor triplet-repeat polymorphism. We tested nine female carriers and nine unrelated normal females.

\section{Results and discussion}

In the two-point linkage analysis, the highest LOD score of 3.55 was obtained at marker loci DXS1111, 
Fig. 2a, b Molecular analysis of human EDA. a LOD scores with chromosome Xq12-q13.1 microsatellite markers. b DNA sequence electropherogram from individual IV:3, IV:4, V:1, and $\mathrm{V}: 2$. Female carrier $(\mathrm{IV}: 4)$ has $\mathrm{C} / \mathrm{G}$ heterozygosity at bp193 in exon 1 and her healthy husband (IV:3) is a C

hemizygote at the same position. Their affected son (V:1) shows G193 and their unaffected daughter $(\mathrm{V}: 2)$ is a C193 homozygote a

\begin{tabular}{lccccccc}
\hline \multirow{2}{*}{ locus } & \multicolumn{7}{c}{ LOD score at recombination fraction $(\theta)$} \\
\cline { 2 - 8 } & 0 & 0.01 & 0.05 & 0.1 & 0.2 & 0.3 & 0.4 \\
\hline DXS991 & -infini & 0.67 & 1.21 & 1.31 & 1.18 & 0.88 & 0.48 \\
DXS1213 & -infini & -3.34 & -1.39 & -0.67 & -0.15 & 0.01 & 0.03 \\
DXS1111 & 3.55 & 3.49 & 3.27 & 2.98 & 2.34 & 1.64 & 0.85 \\
DXS135 & 2.04 & 2.01 & 1.85 & 1.66 & 1.25 & 0.83 & 0.4 \\
DXS1690 & 2.65 & 2.6 & 2.43 & 2.21 & 1.73 & 1.2 & 0.62 \\
DXS1689 & 3.55 & 3.49 & 3.27 & 2.98 & 2.34 & 1.64 & 0.85 \\
DXS8107 & 2.65 & 2.6 & 2.43 & 2.21 & 1.73 & 1.2 & 0.62 \\
DXS8101 & 3.55 & 3.49 & 3.27 & 2.98 & 2.34 & 1.64 & 0.85 \\
DXS559 & 1.74 & 1.71 & 1.6 & 1.44 & 1.12 & 0.76 & 0.38 \\
DXS1124 & - -infini & -1.61 & -0.33 & 0.13 & 0.42 & 0.42 & 0.27 \\
DXS986 & -infini & -3.06 & 1.09 & 0.36 & 0.18 & 0.3 & 0.22 \\
\hline
\end{tabular}

b Female carrier

C/G Heterozygosis (193)

Normal male

C (193)

$\sqrt{ }$

AC CTAG AGTTGNGCTCGGAGTT

AC C TAG AGTTGCGCTCGGAGTT

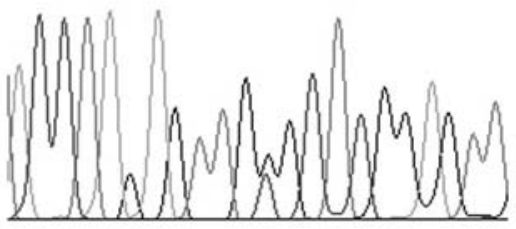

Affected male

$\mathrm{C}>\mathrm{G}$ mutation (193)

$\sqrt{ }$

AC CTAG AGTTGGGCTCGGAGTT

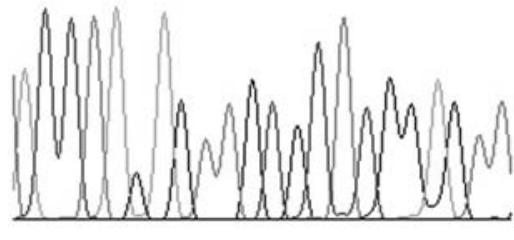

Normal female

$\mathrm{C} / \mathrm{C}$ homozygosis (193)

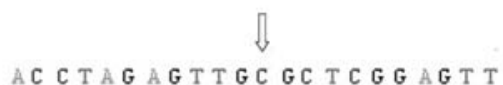

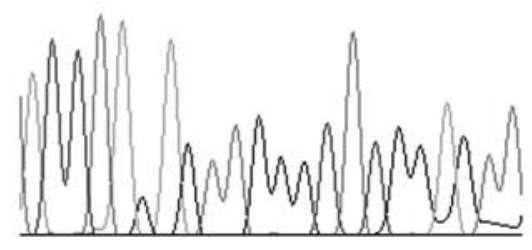

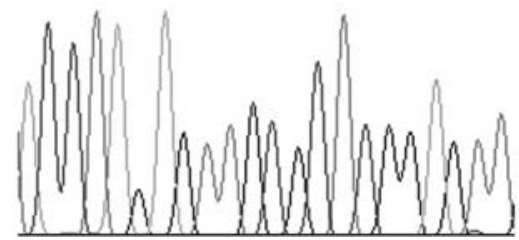

DXS1689, and DXS8101. The adjacent marker also showed an LOD score of $>2$ (Fig. 2a). By haplotype analysis of the pedigree (Appendix), the affected locus was confined to a $<6.48$-cM interval between $D X S 1124$ and $D X S 1213$ at Xq12-q13.1.

Based on previous studies (Kere et al. 1996), we selected $E D A$ as the candidate gene and sequenced all eight exons in two affected males, two female carriers, one normal male, and one normal female subjects. We found a novel missense mutation c. $193 \mathrm{C}>\mathrm{G}$ in exon 1 of $E D A$, and found the mutation segregating with affected or carrier status in the other family members recruited for the larger linkage study (Fig. 2b). Exon 1 of $E D A$ was also sequenced from 90 unrelated normal Chinese Han individuals, 45 females and 45 males, without detecting any $\mathrm{G}$ alleles at bp193 of $E D A$. This mutation will cause glycine to be substituted by arginine at the 65 th residue of ectodysplasin. The R65G mutation is on the edge of the transmembrane domain of ectodysplasin, and changes the isoelectric point and may alter the local structure of EDA.

Analysis of the pattern of $\mathrm{X}$-chromosome inactivation showed that $33 \%$ of nine female carriers had a skewed pattern, while the others showed a random 


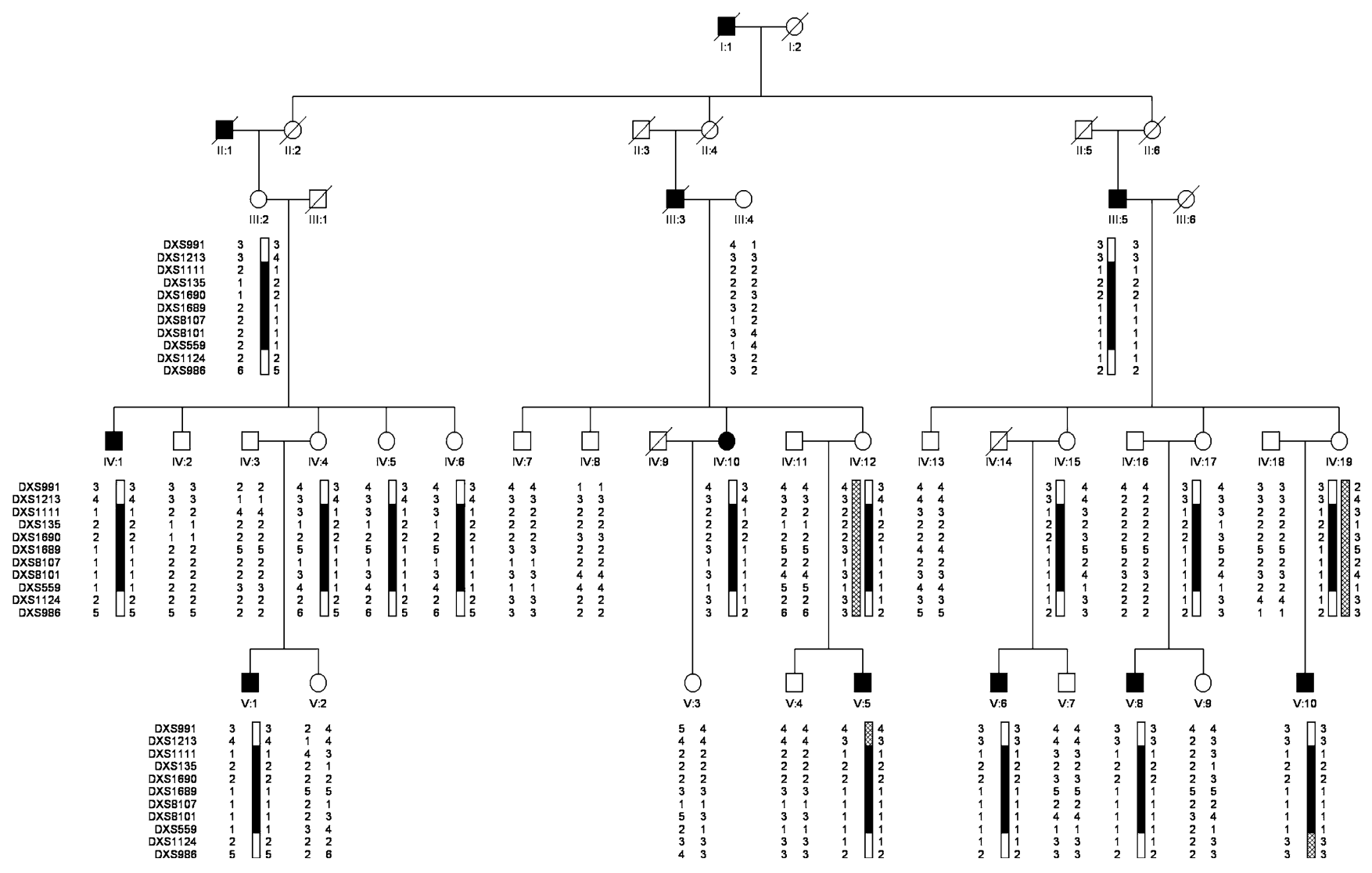

Appendix Haplotype analysis in the family. Marker order was determined from the Généthon sex-averaged genetic map, the CHLC sex-averaged genetic map, and the Genome Database. Open symbols indicate unaffected individuals, blackened symbols indicate the affected individuals, squares indicate men, and circles indicate women. The blackened bars are the seven contiguous-marker disease-linked haplotypes shared by all patients and female carriers. Recombinations of maternal alleles on V:5 and V:10 suggested the boundary of disease-linked haplotypes
$\mathrm{X}$-chromosome inactivation manner. III:2 and IV:10 showed extremely skewed ( $>90 \%$ ) methylation of one $\mathrm{X}$ chromosome and IV:19 revealed moderately skewed (80-90\%) methylation. Skewed inactivation of the X chromosome bearing the wild type EDA gene may increase the proportion of mutated protein in the symptomatic carrier IV:10.

Our results indicate that the novel missense mutation in $E D A$ is associated with tooth agenesis. Though the family inherited unique hypodontia in X-linked manner without abnormalities of other ectodermal organs, our genetic study is consistent with X-linked HED. So, we think the unique hypodontia phenotype may be a clinical subtype of X-linked HED. Ectodysplasin may play a different role in the early development of teeth than with the other ectodermal organs. The Arg65Gly mutation may have an effect in one of several ways: (1) affecting the overall structure of ectodysplasin; (2) abnormal transmembrane trafficking and proteolytic cleavage; (3) gain of function due to a novel interaction site; (4) interaction of ectodysplasin with $E D A R$. Further in vivo expression and functional characterization of the mutated protein may advance our understanding.
Acknowledgements We sincerely thank all the subjects participating in this study, and all the medical staff involved in the diagnosis and sample collection. This work was supported by grants from the national 973 and 863 programs, the National Natural Science Foundation of China, and the Shanghai Municipal Commission for Science and Technology.

\section{References}

Bayes M, Hartung AJ, Ezer S, Pispa J, Thesleff I, Srivastava AK, Kere J (1998) The anhidrotic ectodermal dysplasia gene (EDA) undergoes alternative splicing and encodes ectodysplasin-A with deletion mutations in collagenous repeats. Hum $\mathrm{Mol}$ Genet 7(11):1661-1669

Darwin CR (1875) The variation of animals and plants under domestication, 2nd edn. John Murray, London

Ezer S, Bayes M, Elomaa O, Schlessinger D, Kere J (1999) Ectodysplasin is a collagenous trimeric type II membrane protein with a tumor necrosis factor-like domain and co-localizes with cytoskeletal structures at lateral and apical surfaces of cells. Hum Mol Genet 8(11):2079-2086

Kere J, Srivastava AK, Montonen O, Zonana J, Thomas N, Ferguson B, Munoz F, Morgan D, Clarke A, Baybayan P, Chen EY, Ezer S, Saarialho-Kere U, de la Chapelle A, Schlessinger D (1996) X-linked anhidrotic (hypohidrotic) ectodermal dysplasia is caused by mutation in a novel transmembrane protein. Nat Genet 13(4):409-416 
Monreal AW, Zonana J, Ferguson B (1998) Identification of a new splice form of the EDAl gene permits detection of nearly all $\mathrm{X}$-linked hypohidrotic ectodermal dysplasia mutations. Am J Hum Genet 63(2):380-389

Pinheiro M, Freire-Maia N (1994) Ectodermal dysplasias: a clinical classification and a causal review. Am J Med Genet 53(2):153162
Tucker AS, Headon DJ, Schneider P, Ferguson BM, Overbeek P, Tschopp J, Sharpe PT (2000) Edar/Eda interactions regulate enamel knot formation in tooth morphogenesis. Development 127(21):4691-4700 